\title{
Prevalência de doenças neurodegenerativas em idosos atendidos em um centro de referência em Belém - PA
}

\author{
Prevalence of neurodegenerative diseases in elderly people at a reference center in Belém - PA \\ Prevalencia de enfermedades neurodegenerativas en ancianos en un centro de referencia en Belém \\ - PA
}

Recebido: 11/01/2022 | Revisado: 19/01/2022 |Aceito: 21/01/2022 | Publicado: 23/01/2022

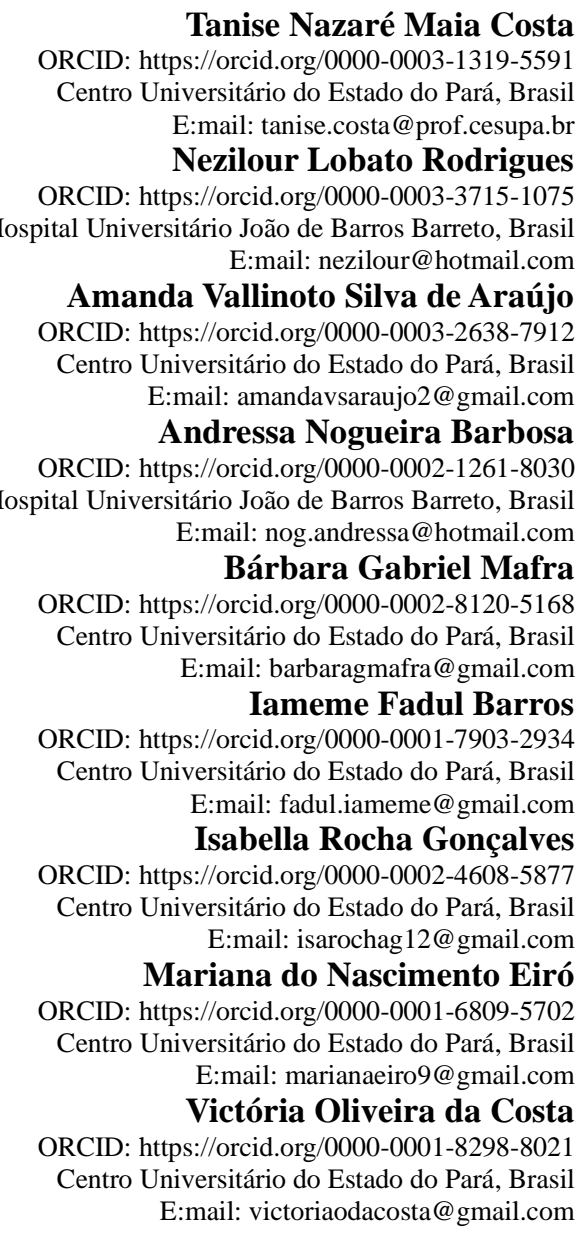

\begin{abstract}
Resumo
O estudo objetivou identificar a prevalência dessas doenças em idosos atendidos em uma unidade de saúde, em Belém - Pará. A pesquisa transversal, observacional e estatístico-descritivo com 470 prontuários de idosos observou prevalência de 7,9\% de Depressão, não condizente com outros estudos que estimam prevalência maior, não foi observado associação com maior idade com distribuição semelhante entre os sexos. Em relação às demências e doença de Parkinson, também não houve alteração relacionada aos sexos, a distribuição entre as faixas etárias foi maior naqueles acima de 70 anos. Conclui-se que o principal fator associado às três condições clínicas foi o envelhecimento, sendo fundamental permitir que seja saudável e bem-sucedido, com controle de fatores de risco para doenças neurodegenerativas e bom suporte social e familiar.
\end{abstract}

Palavras-chave: Estudos transversais; Doenças neurodegenerativas; Idoso.

\section{Abstract}

The study aims to identify the prevalence of these diseases in elderly people cared for at a health unit in Belém - Pará. The cross-sectional, observational and descriptive statistical study with 470 elderly medical records observed a prevalence of $7.9 \%$ of Depression, not consistent with others studies that estimate higher prevalence, no association 
with older age with similar distribution between genders was observed. Regarding dementia and Parkinson's disease, there was also no change related to gender, the distribution between age groups was greater in those over 70 years old. It is concluded that the main factor associated with the three clinical conditions was aging, being essential to allow it to be healthy and successful, with control of risk facts for neurodegenerative diseases and good social and family support.

Keywords: Cross-sectional studies; Neurodegenerative diseases; Aged.

\section{Resumen}

El estudio tuvo como objetivo identificar la prevalencia de estas enfermedades en ancianos atendidos en una unidad de salud en Belém - Pará. Una investigación transversal, observacional y descriptivo-estadística con 470 prontuarios de ancianos observó una prevalencia del 7,9\% de Depresión, lo cual no concuerda con otros estudios que estiman mayor prevalencia, no se observó asociación con mayor edad con distribución similar entre sexos. En relación a las demencias y la enfermedad de Parkinson, tampoco hubo cambio relacionado con el género, la distribución entre grupos de edad fue mayor en los mayores de 70 años. Se concluye que el principal factor asociado a las tres condiciones clínicas fue el envejecimiento, siendo fundamental permitirles estar sanos y exitosos, con control de factores de riesgo para enfermedades neurodegenerativas y buen apoyo social y familiar.

Palabras clave: Estudios transversales; Enfermedades neurodegenerativas; Anciano.

\section{Introdução}

Fenômeno mundial que tem ocorrido nos últimos anos, com maior impacto nos países em desenvolvimento, o envelhecimento populacional apresenta íntima ligação entre os processos de transição demográfica e epidemiológica. Diversos fatores influenciam direta e indiretamente neste processo, sendo dois reconhecidamente de maior peso: redução da natalidade e aumento da expectativa de vida, a partir da redução de taxas de mortalidade (Oliveira, 2019).

O Brasil, que há anos tem vivenciado efeitos de tais transições, tem apresentado progressivamente aumento da população idosa devido a elevação da expectativa de vida, melhores condições sociais e econômicas, além do aumento da prevalência de doenças crônicas não transmissíveis (DCNT) (Mourão et al., 2016).

A associação entre as DCNT e alterações naturais do envelhecimento tornam essa população mais susceptível ao processo de fragilização e dependência, de tal maneira a contribuir para um real problema de saúde pública (Mourão et al., 2016; Schmidt et al, 2011).

Conforme ocorre aumento da expectativa de vida a parcela populacional de idosos aumenta, da mesma maneira que as DCNT mais prevalentes nesta população aumentam proporcionalmente, entre essas doenças destacam-se a depressão, demências (Gullich et al., 2016; Santos et al., 2020).

A depressão é um transtorno crônico, muitas vezes recorrente e potencialmente incapacitante, que onera o sistema público de saúde, causando impacto direto no cotidiano familiar (Lima et al, 2016). Atualmente a depressão é considerada um problema de saúde pública, afetando no mundo aproximadamente 154 milhões de indivíduos, ao avaliarmos a prevalência na população geriátrica encontramos cerca de $15 \%$ de pessoas com depressão ou sintomas depressivos clinicamente significantes (Gullich et al., 2016).

No transtorno depressivo diversas alterações psicopatológicas ocorrem, podendo o quadro clínico (sintomatologia, gravidade, curso e prognóstico) diferir entre as pessoas que apresentam tal diagnóstico. Entre tais sintomas pode-se observar humor deprimido e anedonia, acompanhados de irritabilidade, sensação subjetiva de cansaço e/ou fadiga, alterações do sono e apetite, desinteresse, lentificação de pensamentos ou dificuldade para concentrar-se, entre outros. Tais sintomas podem gerar ideias de fracasso, prejudicando de forma expressiva a qualidade de vida do idoso (Lima et al, 2016).

O grupo que abrange os diversos tipos de demências, ou Transtorno Neurocognitivo Maior (TNCM), é uma das principais causas de comprometimento funcional das atividades de vida diária e declínio cognitivo com prejuízo de autonomia, impactando diretamente na qualidade de vida do idoso (Santos et al., 2020; Confortin et al, 2019). A demência por doença de Alzheimer (DA) é uma síndrome neurodegenerativa progressiva e crônica, sendo a etiologia mais comum correspondendo 
cerca de 60\% a 70\% dos casos. Além da DA, outras causas importantes são demência vascular, demência por corpos de Lewy, demência fronto-temporal, entre outros (Lini et al, 2016).

A idade avançada é um dos fatores de risco já conhecidos e não modificáveis, alguns fatores de risco modificáveis têm sido estudados com o intuito de prevenir o surgimento da doença. Publicação do The Lancet estudou os fatores de risco modificáveis, entre eles: baixa escolaridade, perda auditiva, menor renda, baixa escolaridade, presença de depressão, isolamento social, sedentarismo, tabagismo e poluição do ar (Livingston et al, 2020).

Outra entidade associada a alterações neurodegenerativas cerebrais, é a doença de Parkinson em que ocorre degeneração de células da camada ventral da parte compacta da substância nigra e do locus ceruleus, e que conforme a sua evolução podem evoluir para TNCM em até 80\% dos casos (Freitas, 2017; Tang et al, 2017). É definida clinicamente pela presença de bradicinesia e pelo menos uma característica motora cardinal adicional (rigidez ou tremor de repouso). O início dos sintomas motores é geralmente unilateral e a assimetria persiste em toda a doença (Poewe et al, 2017).

Em vista do exposto, entende-se que o processo de envelhecimento populacional traz consigo o aumento de doenças crônico-degenerativas e, no contexto das alterações cognitivas do paciente idoso, cita-se a Depressão, as Demências e a Doença de Parkinson com grande importância no referido tema. O presente estudo objetiva identificar a prevalência de tais doenças (Depressão, Demência e Doença de Parkinson) em idosos, assim como sua distribuição entre sexo e idade, atendidos em uma unidade municipal de saude, Casa de Saude do Idoso, na cidade de Belém, estado do Pará.

\section{Metodologia}

Trata-se de um estudo transversal, observacional e estatístico-descritivo, que tem como base a análise de prontuários de 470 idosos, de ambos os sexos, em acompanhamento na Casa de Saude do Idoso, na cidade de Belém - PA. Este estudo foi aprovado pelo Comitê de Ética em Pesquisa do Centro Universitário do Estado do Pará (3.426.166).

O formulário de coleta, de autoria própria, contém informações acerca do sexo, idade e presença de Transtorno Neurocognitivo Maior e Depressão. A presença de tais patologias foi considerada mediante diagnóstico prévio registrado no prontuário. Foram excluídos do estudo pacientes cujos prontuários possuem dados inconclusivos.

Os dados foram tratados pelo programa Microsoft Office Excel 2017. A análise estatística foi realizada no programa SPSS (Statistical Package for the Social Sciences). Para verificar a existência de associação entre TNCM, Depressão e Doença de Parkinson e as variáveis independentes sexo e idade, foi aplicado o teste de qui-quadrado de aderência. A significância estatística foi aceita ao nível de 95\% (p-valor <0,05).

Foi utilizado o Termo de Compromisso de Utilização de Dados (TCUD) para obtenção das informações contidas em prontuários.

\section{Resultados}

Dos 470 indivíduos estudados, 358 (76,2\%) eram do sexo feminino e 112 (23,8\%), do sexo masculino. A idade variou de 60 a 117 anos, com média de 76,65 (DP=7,87). Quanto à faixa etária, 102 idosos apresentavam idade entre 60 e 69 anos e 368 idosos apresentavam 70 anos ou mais.

A prevalência de TNCM foi de $16,2 \%$, sendo semelhante entre os sexos $(\mathrm{p}=0,97)$. Ao avaliar a prevalência de acordo com a faixa etária, foi identificado predomínio dessa patologia em indivíduos com idade igual ou superior a 70 anos $(p=$ $<0,00001$ ) (Tabela 1). Nos idosos entre 60 e 69 anos, não houve mulheres com TNCM; enquanto observou-se 1 homem com esse diagnóstico. Ao comparar os sexos em idosos com idade igual ou superior a 70 anos, não houve diferença significativa $(\mathrm{p}=0,87)($ Tabela 2$)$. 
Tabela 1. Frequência de TNCM por faixa etária e gênero.

\begin{tabular}{llll}
\hline Variável & n & TNCM & p-valor \\
\hline Mulheres & 358 & $58(16,2 \%)$ & 0,97 \\
Homens & 112 & $18(16,1 \%)$ & \\
$60-69$ anos & 102 & $1(1 \%)$ & $<0,00001$ \\
$\geq 70$ anos & 368 & $75(20,3 \%)$ & \\
\hline
\end{tabular}

Fonte de protocolo de pesquisa.

Tabela 2. Frequência comparativa de TNCM por faixa etária e gênero.

\begin{tabular}{lllll}
\hline Variável & & n & TNCM & p-valor \\
\hline \multirow{2}{*}{$60-69$ anos } & Mulheres & 76 & $0(0 \%)$ & \\
& Homens & 26 & $1(3,8 \%)$ & \\
$\geq 70$ anos & Mulheres & 282 & $58(20,5 \%)$ & 0,87 \\
& Homens & 86 & $17(19,7 \%)$ & \\
\hline
\end{tabular}

Fonte de protocolo de pesquisa.

Quanto à Depressão, a prevalência foi de 7,9\%, sendo 7,3\% para o sexo feminino e 9,8\% para o sexo masculino (p= 0,38). Ao avaliar as faixas etárias, a prevalência foi semelhante $(\mathrm{p}=0,97)$ (Tabela 3). Quando analisado por faixa etária, também foi observada semelhança na prevalência desse diagnóstico entre os sexos (Tabela 4).

Tabela 3. Frequência de Depressão por faixa etária e gênero.

\begin{tabular}{llll}
\hline Variável & n & Depressão & p-valor \\
\hline Mulheres & 358 & $26(7,2 \%)$ & 0,38 \\
Homens & 112 & $11(9,8 \%)$ & \\
$60-69$ anos & 102 & $9(8,8 \%)$ & 0,97 \\
$\geq 70$ anos & 368 & $28(7,6 \%)$ & \\
\hline
\end{tabular}

Fonte de protocolo de pesquisa.

Tabela 4. Frequência comparativa de Depressão por faixa etária e gênero.

\begin{tabular}{lllll}
\hline Variável & & n & Depressão & p-valor \\
\hline \multirow{2}{*}{$60-69$ anos } & Mulheres & 76 & $6(7,8 \%)$ & 0,57 \\
& Homens & 26 & $3(11,5 \%)$ & \\
$\geq 70$ anos & Mulheres & 282 & $20(7,0 \%)$ & 0,49 \\
& Homens & 86 & $8(9,3 \%)$ & \\
\hline
\end{tabular}

Fonte de protocolo de pesquisa.

A prevalência de Doença de Parkinson foi de 5,7\%. 4,7\% das mulheres e 8,7\% dos homens apresentaram esse diagnóstico $(p=0,09)$. Quando comparadas as faixas etárias, não houve diferença significativa $(p=0,64)($ Tabela 5). Ao analisar a prevalência de Doença de Parkinson por sexo em cada faixa etária, os resultados foram semelhantes para o grupo de 60 a 69 anos $(p=0,44)$ e para os idosos com idade igual ou superior 70 anos $(p=0,13)$ (Tabela 6). 
Tabela 5. Frequência de Doença de Parkinson por faixa etária e gênero.

\begin{tabular}{llll}
\hline Variável & n & Doença de Parkinson & p-valor \\
\hline Mulheres & 358 & $17(4,7 \%)$ & 0,09 \\
Homens & 112 & $10(8,9 \%)$ & \\
$60-69$ anos & 102 & $5(4,9 \%)$ & 0,64 \\
$\geq 70$ anos & 368 & $22(5,9 \%)$ & \\
\hline
\end{tabular}

Fonte: Protocolo de pesquisa.

Tabela 6. Frequência comparativa de Doença de Parkinson por faixa etária e gênero.

\begin{tabular}{|c|c|c|c|c|}
\hline Variável & & $\mathbf{n}$ & Doença de Parkinson & p-valor \\
\hline \multirow[t]{2}{*}{$60-69$ anos } & Mulheres & 76 & $3(3,9 \%)$ & \multirow{2}{*}{0,57} \\
\hline & Homens & 26 & $2(7,6 \%)$ & \\
\hline \multirow{2}{*}{$\geq 70$ anos } & Mulheres & 282 & $14(4,9 \%)$ & \multirow{2}{*}{0,49} \\
\hline & Homens & 86 & $8(9,3 \%)$ & \\
\hline
\end{tabular}

Fonte: Protocolo de pesquisa.

Ao analisar a prevalência simultânea de TNCM, Depressão e Doença de Parkinson, observou-se que 0,85\% dos idosos apresentavam o diagnóstico de TNCM e Depressão, 0,63\% apresentavam TNCM e Doença de Parkinson, 0,42\% apresentavam Depressão e Doença de Parkinson e nenhum idoso obtinha os três diagnósticos simultâneos.

\section{Discussão}

No presente estudo, observou-se uma prevalência de 7,9\% de Depressão entre os idosos estudados. Outros estudos apontam uma prevalência de Depressão maior no Brasil entre idosos não institucionalizados de $3 \%$ a $15 \%$ e a presença de sintomas depressivos clinicamente significativos variaram de $13 \%$ a 39\% (Silva et al., 2019).

Um estudo realizado com 151 idosos observou que 21,2\% desses apresentavam esse diagnóstico (Borges \& Dalmolin, 2012). A reduzida prevalência de Depressão, em relação aos demais estudos, pode ser decorrente de diferenças socioeconômicas e demográficas associadas a fatores de risco.

Dentre os fatores de risco associados à Depressão, destacam-se: baixa escolaridade, baixo nível de atividade social, pior estado funcional, diagnóstico de Hipertensão Arterial Sistêmica e Acidente Vascular Encefálico. Em dois estudos brasileiros e um chines a depressão foi associada ao sexo feminino (Nóbrega et al., 2015; Tsai et al., 2005). Estudo português encontrou maior prevalência entre idosos do sexo masculino, viúvos e institucionalizados, associando neste trabalho a influência do ambiente e suporte emocional em que tais idosos estão inseridos (Matias et al., 2016). Nesta avaliação realizada no presente estudo não foi observada uma diferença significativa entre os sexos.

Acerca da faixa etária, não foi observada diferença significativa. Um estudo chines relacionou a Depressão com a maior idade (Ku \& Liu, 2006; Tsai et al., 2005). Em contrapartida, um estudo realizado na Holanda associou este diagnóstico com idosos de idade inferior a 80 anos (Smalbrugge et al., 2006). Quanto ao cenário de Depressão, no presente estudo não houve diferença significativa entre as faixas etárias e os sexos.

Demência ou TNCM apresenta como as principais causas o grupo de etiologias neurodegenerativas e cerebrovasculares, apresentando em diversos estudos aumento conforme o envelhecimento (Dalgalarrondo, 2018). Neste estudo a prevalência de Transtorno Neurocognitivo Maior não se demonstrou diferente conforme os sexos, porém ocorreu diferença relevante entre as faixas-etárias, sendo mais predominante em pacientes com mais de 70 anos, situação que se assemelha a um estudo realizado com 196 idosos no Rio Grande do Sul (Lini et al, 2016). 


\section{Conclusão}

O envelhecimento populacional tem trazido desafios para a sociedade, equipes médicas, familiares e cuidadores. Com tal processo o número de idosos tem aumentado constantemente, acompanhado assim do aumento proporcional de doenças e condições clínicas que sejam prevalentes nesta faixa etária, principalmente as doenças crônicas não transmissíveis. Entre tais doenças, no âmbito da geriatria, ganha-se importante destaque aquelas que possam contribuir para a perda de autonomia e de independência: depressão, transtorno neurocognitivo maior e doença de Parkinson são exemplos.

Políticas de saúde pública devem ser traçadas com base nestes dados presentes na literatura que suportam que o diagnóstico precoce de certas condições e seus tratamentos curativos, de reabilitação ou paliativos agregam qualidade de vida e redução de custos ao sistema.

Conforme avaliado neste estudo o principal fator associado às três condições clínicas na nossa população foi o próprio envelhecimento. Permitir um envelhecimento saudável e bem-sucedido, com controle de fatores de risco para doenças neurodegenerativas, bom suporte social e familiar é de suma importância para que essa prevalência diminua, de tal maneira que essa população goze de melhores condições de vida.

Mais estudos devem ser realizados a fim de avaliarem a associação entre as três condições analisadas e como essas possíveis associações contribuem para o processo de senilidade.

\section{Referências}

Borges, D. T., \& Dalmolin, B. M. (2012). Depressão em idosos de uma comunidade assistida pela Estratégia de Saúde da Família em Passo Fundo, RS. Revista Brasileira de Medicina de Família e Comunidade, 7(23), 75-82.

Confortin, S. C., Meneghini, V., Ono, L. M., Garcia, K. C., Schneider, I. J. C., d’Orsi, E., \& Barbosa, A. R. (2019). Indicadores antropométricos associados à demência em idosos de Florianópolis-SC, Brasil: Estudo EpiFloripa Idoso. Ciência \& Saúde Coletiva, 24, $2317-2324$.

Dalgalarrondo, P. (2018). Psicopatologia e semiologia dos transtornos mentais. Artmed Editora.

Freitas, E. V. D., Py, L., Cançado, F. A. X., Doll, J., \& Gorzoni, M. L. (2017). Tratado de geriatria e gerontologia. Editora Guanabara Koogan.

Gullich, I., Duro, S. M. S., \& Cesar, J. A. (2016). Depressão entre idosos: um estudo de base populacional no Sul do Brasil. Revista Brasileira de Epidemiologia, 19, 691-701.

Ku, Y. C., Liu, W. C., \& Tsai, Y. F. (2006). Prevalence and risk factors for depressive symptoms among veterans home elders in Eastern Taiwan. International Journal of Geriatric Psychiatry: A journal of the psychiatry of late life and allied sciences, 21(12), 1181-1186.

Lima, A. M. P., Ramos, J. L. S., Bezerra, I. M. P., Rocha, R. P. B., Batista, H. M. T., \& Pinheiro, W. R. (2016). Depressão em idosos: uma revisão sistemática da literatura. Revista de Epidemiologia e Controle de Infecção, 6(2), 97-103.

Lini, E. V., Lima, A. P. D., Giacomazzi, R. B., Doring, M., \& Portella, M. R. (2016). Prevalência e fatores associados aos sintomas sugestivos de demência em idosos. Cien. Cogn, 21(2), 189-97.

Livingston, G., Huntley, J., Sommerlad, A., Ames, D., Ballard, C., Banerjee, S., \& Mukadam, N. (2020). Dementia prevention, intervention, and care: 2020 report of the Lancet Commission. The Lancet, 396(10248), 413-446.

Matias, A. G. C., Fonsêca, M. D. A., Gomes, M. D. L. D. F., \& Matos, M. A. A. (2016). Indicadores de depressão em idosos e os diferentes métodos de rastreamento. Einstein, 14, 6-11.

Mourão, L. F., Xavier, D. A. N., Neri, A. L., \& Luchesi, K. F. (2016). Estudo da associação entre doenças crônicas naturais do envelhecimento e alterações da deglutição referidas por idosos da comunidade. Audiology-Communication Research, 21.

Nóbrega, I. R. A. P. D., Leal, M. C. C., Marques, A. P. D. O., \& Vieira, J. D. C. M. (2015). Fatores associados à depressão em idosos institucionalizados: revisão integrativa. Saúde em Debate, 39, 536-550.

Oliveira, A. S. (2019). Transição demográfica, transição epidemiológica e envelhecimento populacional no Brasil. Hygeia-Revista Brasileira de Geografia Médica e da Saúde, 15(32), 69-79.

Poewe, W., Seppi, K., Tanner, C. M., Halliday, G. M., Brundin, P., Volkmann, J., \& Lang, A. E. (2017). Parkinson disease. Nature reviews Disease primers, $3(1), 1-21$.

Santos, C. D. S. D., Bessa, T. A. D., \& Xavier, A. J. (2020). Fatores associados à demência em idosos. Ciência \& Saúde Coletiva, $25,603-611$.

Schmidt, M. I., Duncan, B. B., Silva, G. A., Menezes, A. M., Monteiro, C. A., \& Barreto, S. M. (2011). Doenças crônicas não-transmissíveis no Brasil: carga e desafios atuais. 
Research, Society and Development, v. 11, n. 2, e22911225627, 2022

(CC BY 4.0) | ISSN 2525-3409 | DOI: http://dx.doi.org/10.33448/rsd-v11i2.25627

Silva, P. O., Aguiar, B. M., Vieira, M. A., Costa, F. M. D., \& Carneiro, J. A. (2020). Prevalência de sintomas depressivos e seus fatores associados em idosos atendidos por um centro de referência. Revista Brasileira de Geriatria e Gerontologia, 22.

Smalbrugge, M., Jongenelis, L., Pot, A. M., Eefsting, J. A., Ribbe, M. W., \& Beekman, A. T. (2006). Incidence and outcome of depressive symptoms in nursing home patients in the Netherlands. The American Journal of Geriatric Psychiatry, 14(12), 1069-1076.

Tang, Y. et al. (2017). Diferenças metabólicas cerebrais associadas ao comprometimento cognitivo na doença de Parkinson. PLoS ONE, 11 , e0152716.

Tsai, Y. F., Chung, J. W., Wong, T. K., \& Huang, C. M. (2005). Comparison of the prevalence and risk factors for depressive symptoms among elderly nursing home residents in Taiwan and Hong Kong. International Journal of Geriatric Psychiatry: A journal of the psychiatry of late life and allied sciences, 20(4), 315-321. 